\title{
Simulation of rain fade on arbitrary microwave link networks by the downscaling and interpolation of rain radar data
}

\author{
Kevin S. Paulson ${ }^{1}$ and Xaiobei Zhang ${ }^{1}$ \\ Received 10 June 2008; revised 23 January 2009; accepted 5 February 2009; published 2 April 2009.
}

[1] To predict the Quality of Service at a node in heterogeneous networks of line-of-sight, terrestrial, microwave links requires knowledge of the spatial and temporal statistics of rain over scales of a few meters to tens or hundreds of kilometers, and over temporal periods as short as $1 \mathrm{~s}$. Meteorological radar databases provide rain rate maps over areas with a spatial resolution as fine as a few hundred meters and a sampling period of 2 to 15 min. Such two-dimensional, rain rate map time series would have wide application in the simulation of rain scatter and attenuation of arbitrary millimeter-wave radio networks, if the sampling period were considerably shorter, i.e., of the order of $10 \mathrm{~s}$ or less, and the integration volumes smaller. This paper investigates a stochastic-numerical method to interpolate and downscale rain rate field time series to shorter sampling periods and smaller spatial integration areas, while conserving the measured and expected statistics. A series of radar derived rain maps, with a 10 min sample period, are interpolated to $10 \mathrm{~s}$. The statistics of the interpolated-downscaled data are compared to fine-scale rain data, i.e., $10 \mathrm{~s}$ rain gauge data and radar data with a $300-\mathrm{m}$ resolution. The interpolated rain map time series is used to predict the fade duration statistics of a microwave link, and these are compared to a published and ITU-R model.

Citation: Paulson, K. S., and X. Zhang (2009), Simulation of rain fade on arbitrary microwave link networks by the downscaling and interpolation of rain radar data, Radio Sci., 44, RS2013, doi:10.1029/2008RS003935.

\section{Introduction}

[2] Terrestrial, line-of-sight, microwave telecommunications links experience attenuation due to rain. At frequencies above $10 \mathrm{GHz}$ this is the dominant fade mechanism and (with mechanical failure) is almost exclusively the cause of outage. Outage has a complex definition stemming from ITU-R recommendations G.826 (1999) and G.828 (2000) and the F recommendations that are derived from them (e.g., F.1491). Modern digital radio systems broadcast a bit stream divided into blocks, e.g., a typical SDH STM-1 system might have 801 bits per block and transmit 192,000 blocks per second. If any bit within a block is transmitted incorrectly, then the block is termed "errored". A Severely Errored Second (SES) occurs in any second where 30\% or more of the blocks were errored. An outage is defined as the period between the first of ten consecutive SES until the first of ten consecutive non-SES. Traditionally,

\footnotetext{
${ }^{1}$ Department of Engineering, University of Hull, Kingston upon Hull, UK.

Copyright 2009 by the American Geophysical Union. 0048-6604/09/2008RS003935
}

links are specified to have an outage period caused by rain fading not exceeding some small percentage of an average year, usually $0.01 \%$ or $0.1 \%$ of time, and the rain fade margin is built into the link budget by estimating the rain attenuation exceeded for this time. Many models exist to calculate the fade margin [e.g., COST 210 Management Committee, 1991; COST 235 Management Committee, 1996; ITU-R, 2007a, 2007b]. However, these models are based on available statistics of rain rate measured with a 1-min integration time. These models are adequate for fade-margin calculations for individual long links but probably under estimate the incidence of outage on links shorter than $1 \mathrm{~km}$. They provide only limited guidance on the performance of networks; e.g., $I T U-R$ [2007a] provides some guidance for more complex links such as multihop links and links utilizing route diversity. ITU-R [2003b] also provides some guidance for point-to-multipoint cellular systems.

[3] The Quality of Service (QoS) experienced by a node in a heterogeneous network of microwave links, at a minimum defined by the average annual outage, is currently impossible to predict as it depends upon joint time series of rain fade with a 1-s integration time. Rudimentary models of fade duration exist for individual links, but not for more complicated networks. The 
engineering of fade mitigation techniques such as route diversity or adaptive modulation, similarly require the knowledge of typical time series of rain fade on heterogeneous networks of links [see COST 280 Management Committee, 2005].

[4] It has been demonstrated that joint rain fade on arbitrary networks of microwave links can be calculated from radar derived rain rate fields [e.g., Goddard and Thurai, 1996, 1997; Tan and Pedersen, 2000; Paulson, 2003; Usman, 2005]. These studies and others have derived 10-s integrated rain fade on microwave links from radar measured rain rate maps with 300-m resolution. When meteorological radars are used to scan across near-horizontal planes (PPI scans), they provide nearinstantaneous measurements of radar reflectivity over large areas. Each reflectivity value is a weighted average across a voxel defined by the radar antenna pattern, the angular scan of the radar and the range gates. Measured single- and dual-polarization radar reflectivities may be used to estimate rain rate, either using empirical relationships or theoretical relationships based on parameterized drop size distributions and assumptions about drop shape and terminal velocity [Goddard and Cherry, 1984]. These radar-derived rain rates are averaged over the same voxel as the reflectivity measurement. Large radars, such as CAMRa the Chilbolton Advanced Meteorological Radar, can produce near-instantaneous rain rates averaged over voxels with linear dimensions of a few hundred meters within a range up to $100 \mathrm{~km}$ [Goddard et al., 1994].

[5] Instantaneous joint rain fade is calculated by superimposing the measured rain field over a network of links. The rain fade experienced by each link is calculated by numerical integration of the specific attenuation along the link path. The specific attenuation is derived from the rain rate using a power law $\gamma-R$ relationship [i.e., ITU-R, $2003 a]$. Specific attenuations derived from rain rates are known to include relative errors of $50 \%$ or more depending upon variation in the drop size distribution (DSD). Unlike rain rate, Rayleigh scattering predicts that radar reflectivity and microwave scattering both depend upon the sixth power of drop diameter. Radar-derived specific attenuations are effectively a frequency scaling and are likely to be more accurate than the intermediate rain rates.

[6] The process described above yields instantaneous measurements of joint rain fade. Time series of radar derived rain rate fields can be used to generate joint-fade time series. However, the method is limited by the long temporal sampling period. Radars need to be physically rotated to scan across the area being mapped. The period between consecutive radar images is usually several minutes and can be as long as $15 \mathrm{~min}$. This low temporal sampling rate severely limits the application of the derived rain fade time series.
[7] It has been proposed that joint rain fade and rain scatter interference on heterogeneous link networks of arbitrary geometry and radio parameters could be calculated from simulated rain fields, derived from stochastic models of rainfall [Callaghan, 2004, 2007; Callaghan and Vilar, 2007]. This is currently being considered for adoption into a recommendation by the ITU-R. The evolution of stochastic rain models spanning the wide range of scales necessary for radio system simulation is still in its infancy. At larger scales, stochastic, spatialtemporal rainfall models for hydrological modeling have existed for decades [Wheater et al., 2000]. At the fine scales necessary for radio system simulation, multiscaling and multiplicative cascade models exist. However, the model parameters are based on statistics averaged over a large number of event types and so do not reproduce the rich diversity of rain events, particularly anisotropic events such as squall lines and fronts. Furthermore, the lack of very fine scale rain data, e.g., from integration periods less than $10 \mathrm{~s}$, means these models have not been verified down to the smallest scales.

[8] An alternative method is proposed in this paper, where long time series of spatial rain fields, derived from meteorological radar, are numerically interpolated and downscaled to yield the range of scales necessary for network simulation. The effect is to numerically generate the fine-scale rain fields that may have been measured at times between radar scans. After interpolation and downscaling, the rain field time series considered in this paper has a sampling period of $10 \mathrm{~s}$ or less and can be used as the input to a microwave network simulator. The proposed method has the advantage over purely numerical rain field generation of matching measured rain fields at the radar sample times. The more anisotropic large scale rain variation is conserved from the radar data and other parameters, such as advection, are also retained.

[9] Section 2 describes the database of radar measurements used to develop and verify the proposed method. Section 3 develops the numerical interpolation and downscaling, while section 4 presents evidence verifying the interpolated rain fields and derived rain fade statistics.

\section{Chilbolton Radar Interference Experiment}

[10] The Chilbolton Radar Interference Experiment (CRIE) was a 2-year rain measurement campaign between 1987 and 1989, designed primarily for development and testing of rain scatter interference models as part of the COST 210 project [COST210, 1991]. It aimed to record an unbiased sample of the rain events occurring near Chilbolton, in the south of England, latitude $51^{\circ} 9^{\prime} \mathrm{N}$, longitude $1^{\circ} 26^{\prime} \mathrm{W}$. Rain fields were scanned using the 
Chilbolton Advanced Meteorological Radar, CAMRa, a $25 \mathrm{~m}$ steerable antenna equipped with a $3 \mathrm{GHz}$, DopplerPolarization radar. The climate in the region is temperate maritime with an average annual rain rate exceeded $0.01 \%$ of the time of approximately $30 \mathrm{~mm} / \mathrm{h}$. For the 2-year period the radar was operated on a 28-day duty cycle. A set of near-horizontal (PPI) and vertical (RHI) radar scan were recorded in a 10-min cycle for 9 out of the 28 days. The PPI scans were acquired with an elevation of $1.5^{\circ}$ and covered an area approximately $50^{\circ}$ in azimuth, in $0.25^{\circ}$ steps, centered southwest of the radar. As the scan rate of the radar is 1 degree/s, it took less than $1 \mathrm{~min}$ to complete a PPI scan. Hence, the scan duration is well within the 20-30 min duration for the lifetime of a rain event [Zawadzki, 1973], and each scan represents a good snapshot of the rain field before any significant structural change has taken place.

[11] The resulting database contains 3199 scan sets, and 30590 records of no rain. The radar data were converted into rain rate fields as part of a earlier project [see Usman, 2005], and a summary of the process is now given. Data were collected between the ranges of $4.8 \mathrm{~km}$ and $158 \mathrm{~km}$ from the radar and averaged over $300 \mathrm{~m}$ range intervals. For this paper, only the data between $20 \mathrm{~km}$ and $70 \mathrm{~km}$ are used. This is to avoid sample volumes being within the freezing level and to limit differences in volume averaging due to beam spreading. Each PPI scan has been range corrected, calibrated, and correction made for absorption by atmospheric gasses. Reflectivities below the noise floor of $10 \mathrm{dBZ}$ were assigned the minimum measurable rain rates of $0.05 \mathrm{~mm} / \mathrm{h}$. In subsequent processing this rain rate was used as a flag to indicate a rain rate at or below this level. Scans with no measurements above the noise floor were discarded and recorded as no rain. Comparison of dual polar reflectivities and the rainhail algorithm of Leitao and Watson [1984] has been used to eliminate data points where nonliquid hydrometeors may have influenced reflectivities. Finally, dual polarization reflectivity data were transformed into rain rate fields.

\section{Interpolation and Downscaling of Radar Data}

[12] Interpolation is the process of introducing new rain rate samples, with the same averaging volume as existing measurements, at new times or locations. The new samples should come from the same underlying distribution as existing samples. In contrast, downscaling (also known as dissagregation) transforms a rain rate field with one averaging volume to one with new samples averaged over a smaller volume. The new samples come from a different underlying distribution, typically with greater variance. Both processes rely upon statistical models to constrain statistics that did not exist in the original rain rate fields such as covariance at lags shorter than the finest original sampling interval.

[13] A fundamental statistic of a random field is the moment scaling structure function. Let $S_{q}$ be the $q$ th moment of $R_{\lambda}$ the rain rate measured over spatialtemporal volumes of scale $\lambda$ i.e., $S_{q}(\lambda)=E\left(\left[R_{\lambda}\right]^{q}\right)$. If $S_{q}(\lambda) \propto \lambda^{\xi(q)}$, where $\xi(q)$ does not depend upon $\lambda$, over some range of scales, then the rain rate is said to exhibit scaling. For simple scaling $\xi(q)$ is linear; otherwise, it is known as anomalous or multiscaling. The function $\xi(q)$ yields the multifractal exponents.

[14] Paulson and Zhang [2007] have reported the variation of the moments of rain rate averaged over spatial squares of side $300 \mathrm{~m}$ to $10 \mathrm{~km}$. They also report the moment scaling statistics of point rain rate averaged over temporal periods from $10 \mathrm{~s}$ to $6 \mathrm{~h}$. Spatially averaged rain rate was shown to be scaling from $300 \mathrm{~m}$ to approximately $2 \mathrm{~km}$ and temporal averages over a corresponding interval from $10 \mathrm{~s}$ to $200 \mathrm{~s}$. The lower limits of these scaling ranges correspond to the finest scales present in the measured data rather than a scale break, and it is very likely that these ranges continue to smaller scales. Lovejoy and Schertzer [2006] suggest multiscaling ranges down to dissipation scales around $50 \mathrm{~cm}$.

[15] A range of multiplicative, random-cascade models exist for the generation and downscaling of stochastic fields with specific variation of multifractal exponents [e.g., She and Waymire, 1995; Monin and Yaglom, 1971, 1975; Deidda, 1999]. Interpolation is less well studied. Paulson [2004] demonstrated that rain rate time series derived from rain gauge measurements can be interpolated from 10 -s rain rate averages with a 10 -min sampling period to a 10 -s sampling period, while conserving a range of statistics. The underlying statistical model is that spatial-temporal log rain rate, where raining, is well modeled as a homogenious, isotropic, fractional Brownian field (FBf) with Hurst coefficient $H=1 / 3$ [Paulson, 2002; Callaghan, 2004]. FBfs are a simple scaling, $n$-dimensional extension of Fractional Brownian noise (FBn) [see Mandelbrot and Van Ness, 1968], and have power law spectral density functions, $S(\omega)=\omega^{-\gamma}$, with the power exponent being $\gamma=n+2 H$. A FBf $L(\mathbf{x})$ has Gaussian updates which satisfy the scaling equation:

$$
\frac{L(\mathbf{x})-L(\mathbf{x}+\Delta \mathbf{x})}{\|\Delta \mathbf{x}\|^{H}} \in N\left(0, \sigma^{2}\right)
$$

The same model has been applied to microwave networks in a route-diversity model [Paulson, 2003]. Rain field simulations of this model have been used in other microwave network applications in several studies commissioned by the UK spectrum regulator Ofcom as part of the Spectrum Efficiency Scheme, i.e., SES-2004-7 and SES-2005-4 (http://www.ofcom.org.uk/). Callaghan [2004] has demonstrated that the FBf log rain rate model 
is consistent with published multiscaling exponents of rain rate fields.

[16] In the following sections we examine algorithms for the downscaling and interpolation of rain fields generated from meteorological radar. In particular we will concentrate on the CRIE data set generated by CAMRa. Rain fields measured by radar are typically near-instantaneous measurements of volume averaged rain rate. The rain rates are spatially aggregated but temporally sampled. To produce fine-scale fields for radio network simulation it is necessary to produce rain fields with a much shorter temporal sampling interval, and possibly smaller averaging volumes. Therefore, a combination of interpolation and downscaling is required.

\subsection{Downscaling}

[17] Downscaling introduces new rain rate samples from a different underlying distribution, typically with moments for $q>1$ that increase as the aggregation volume decreases. The variation of these moments as a function of averaging volume needs to be controlled in the downscaling process. A canonical constraint is that the first moment of rain rate is independent of averaging scale. This can be applied to each rain rate sample to yield a microcanonical constraint where downscaling exactly conserves the mean rain rate.

[18] We assume the independence of variation at scales below the radar spatial sampling scale, $\Delta_{x}=300$, on consecutive scans measured $\Delta_{t}=10 \mathrm{~min}$ apart. This will be true as long as $\Delta_{x} / D_{x} \ll \Delta_{t} / D_{t}$, where $D_{x}$ and $D_{t}$ are the spatial and temporal decorrelation distances. For the CRIE data this is true as the spatial resolution is relatively much finer than the interscan period. Even after advection removal, the spatial sampling is an orderof-magnitude finer than the temporal sampling. This allows each measured radar scan to be downscaled independently.

[19] In the current work, downscaling is achieved using the multiplicative cascade algorithm of Deidda [1999, 2000]. Although downscaling could be achieved using a FBf model, e.g., the Local Average Subdivision algorithm of Fenton and Vanmarcke [1990], an explicit multifractal framework exists and is computationally less complex. Also, the multifractal exponents are known for this climate [Paulson and Zhang, 2007]. The Deidda method replaces each rain rate $R_{\Delta}$ averaged over a region of diameter $\Delta$, with four rain rates $R_{\Delta / 2}^{i}=w_{i} R_{\Delta}, i=$ $1,2,3,4$; averaged over a region of diameter $\Delta / 2$. The weights $w_{i}$ are independent and identically distributed (i.i.d) samples from a log-Poisson distribution: $w_{i}=e^{a} \beta^{y}$, where $y$ is an i.i.d sample from a Poisson distribution of mean $c$. The parameters $\beta$ and $c$ are determined by fitting the scaling exponents of the cascade to measured values. The normalizing constant $a=c(1-\beta)$ is determined by the canonical first order constraint, i.e., $E\left(w_{i}\right)=1$. Use of the exponents reported by Paulson and Zhang [2007] yields the parameters $c=10$ and $\beta=1.115$ for region diameters below $2 \mathrm{~km}$. Almost identical variation of the cascade scaling exponents can be produced by suitable choice of $\beta$ while varying the parameter $c$ over five orders of magnitude. As very similar scaling exponents can be produced by a wide range of $c$ and $\beta$ combinations, comparison with other published sources is unhelpful.

\subsection{Interpolation}

[20] Interpolation introduces new rain rate measurements, with the same spatial averaging, at equi-spaced times between two consecutive measured fields. These rain fields should have the same spatial moment scaling statistics as the measured fields and temporal variation consistent with the rain gauge data reported by Paulson and Zhang [2007]. Interpolation will be based on the FBf log rain rate model of Callaghan [2004] and Paulson [2002]. For each pair of scans, a discrete, 3-D, FBf will be calculated that is consistent with the measured scans.

[21] The horizontal advection of rain fields by the ambient wind field is not described by the FBf model. Before interpolation, advection between consecutive scans is removed. Advection is assumed to be a linear translation between radar scans. This assumption would not be valid for large areas or long interscan times but is reasonable for the CRIE data. The advection of the rain field $R\left(\mathbf{x}, t_{1}\right)$ to $R\left(\mathbf{x}, t_{1}+\Delta_{t}\right)$ between the two measurement times is estimated by identifying the two-dimensional spatial lag $\mathbf{y}$ that maximizes the cross correlation, i.e.,

$$
\mathbf{y}_{A} \equiv \max _{\mathrm{y}} E\left(R\left(\mathbf{x}+\mathbf{y}, t_{1}\right) R\left(\mathbf{x}, t_{2}\right)\right)
$$

Each pair of $\log$ rain rate fields, $L_{1}=\ln \left(R\left(\mathbf{x}+\mathbf{y}_{A}, t_{1}\right)\right)$ and $L_{2}=\ln \left(R\left(\mathbf{x}, t_{1}+\Delta_{t}\right)\right)$, is assumed to be from a censored Gaussian fractional Brownian process. The marginal mean $\mu_{L}$ and variance $\sigma_{L}^{2}$ are estimated using a Maximum Likelihood algorithm for censored data: $L>L_{\min }$, where $L_{\min }$ is the smallest measurable log rain rate. Let $A \equiv\left\{A_{i} \in(x, y)\right\}$ be the set of spatial sampling points and $T \equiv\left\{t_{1} \leq T_{i} \leq t_{1}+\Delta_{t}\right\}$ be the equi-spaced interpolation times. The discrete interpolation volume is $V \equiv\{(\mathbf{x}, t): \mathbf{x} \in A, t \in T\}$. Interpolated log rain rate values are calculated using a hierarchical algorithm that introduces new samples separated by distances that decrease exponentially with iteration. The Random Midpoint Displacement algorithm (RMD) of Voss [1985] has been used to refine isotropic FBfs and is applicable to interpolation as existing samples are conserved at each iteration. The RMD algorithm has been applied to the generation of synthetic rain fields by Callaghan [2004, 2007] and Callaghan and Vilar [2007]. The paragraphs below develop an algorithm for asymmetrically sampled FBfs, i.e., fields sampled at different intervals along orthogonal axis directions. 
[22] The RMD takes a FBf evenly sampled at scale $\Delta$ in each dimension and introduces new samples to yield a FBf sampled at scale $\Delta / 2$. Let $L_{\Delta}=\left\{L_{i} ; i=1, \cdots N_{\Delta}\right\}$ be the log rain rate samples in a region of scale $\Delta$ around the interpolate $L_{Y}$ at position $\boldsymbol{Y}$. The interpolated value is chosen to be:

$$
L_{Y}=S\left(L_{\Delta}\right)+\sigma_{\Delta} \varepsilon_{Y}
$$

where $S\left(L_{\Delta}\right)$ and $\sigma_{\Delta}^{2}$ are estimates of the mean and variance of the $L_{Y}$ distribution, while $\varepsilon_{Y}$ is an i.i.d. standard normal sample. For the asymmetric algorithm a linear estimator will be used, i.e., $S\left(L_{\Delta}\right)=a_{0} \mu_{L}+\sum_{i=1}^{N_{\Delta}} a_{i} L_{i}$ where the coefficients $a_{i}$ depend upon the shape and distribution of samples in the scale region and are chosen to satisfy:

$$
\begin{gathered}
E\left(L_{Y}\right)=\mu_{L}, \\
E\left(L_{y} L_{j}\right)=B_{F B f}\left(\delta_{y j}\right) \text { and } \\
E\left(L_{Y}^{2}\right)=B_{F B f}(0) .
\end{gathered}
$$

Here $B_{F B}(\delta)$ is the expected value of the product of two $\log$ rain rates separated by distance $\delta$, given the $\mathrm{FBf}$ assumption. It may be calculated from the marginal distribution and (1), i.e., $B_{\mathrm{FBF}}(\delta)=E\left(L^{2}\right)-\delta^{2 H} \sigma^{2} / 2$. Substituting (3) into the expected values in (4a)-(4c), and given that the $L_{i}$ are independent of $\varepsilon_{Y}$, yields:

$$
\begin{gathered}
E\left(L_{Y}\right)=a_{0} \mu_{L}+\sum_{i=1}^{N_{\Delta}} a_{i} E\left(L_{i}\right)=\sum_{i=0}^{N_{\Delta}} a_{i} \mu_{L}, \\
E\left(L_{y} L_{j}\right)=a_{0} \mu_{L}^{2}+\sum_{i=1}^{N_{\Delta}} a_{i} B_{F B f}\left(\delta_{i j}\right) \text { and } \\
E\left(L_{Y}^{2}\right)=a_{0}^{2} \mu_{L}^{2}+2 a_{0} \mu_{L}^{2} \sum_{i=1}^{N_{\Delta}} a_{i} \\
+\sum_{i=1}^{N_{\Delta}} \sum_{j=1}^{N_{\Delta}} a_{i} a_{j} B_{F B f}\left(\delta_{i j}\right)+\sigma_{\Delta}^{2} .
\end{gathered}
$$

Equations (4a) and (5a) imply that $\sum_{i=0}^{N_{\Delta}} a_{i}=1$. Furthermore, (4b) and (5b) yield a further $N_{\Delta}$ equations linear in $a_{i}$. The coefficients $\left\{a_{i}\right\}$ are found by solving these $N_{\Delta}+1$ linear equations. Once the coefficients $\left\{a_{i}\right\}$ have been found, (4c) and (5c) yield an expression for $\sigma_{\Delta}^{2}$.

[23] For interpolation at the midpoint of regularly spaced samples and with $L_{\Delta}$ taken to be the $N_{\Delta}=2^{n}$ nearest neighbors, Voss's RMD algorithm generated
FBfs using $a_{i}=N_{\Delta}^{-1}$ and noise variance exponentially decreasing with scale. In this case, the interpolation coefficients are calculated once per iteration and the computational cost is negligible. However, the CRIE rain data is more finely sampled in space than in time, where units are decorrelation intervals. Define the second-order moment of measured data as:

$$
B_{L}(\mathbf{y}, \tau) \equiv E(L(\mathbf{x}, t) L(\mathbf{x}+y, t+\tau))
$$

An initial, interpolation scale of $N=2^{m}$ sample units is determined: $B_{L}\left((N+1) \Delta_{x} \mathbf{e}, 0\right) \cong B_{t}\left(0, \Delta_{t}\right)$, where e is a unit vector. To yield samples symmetrically sampled in space and time, $N$-1, equi-spaced, new log rain fields need to be interpolated. This can be achieved in $m$ iterations of an asymmetric RMD (ARMD) using equations (3), (4a)-(4c), and (5a)-(5c). Interpolation regions of diameter $\Delta_{0}=2 N$ in sample units are used in the first iteration and the diameter is halved at each subsequent iteration. The interpolation coefficients $\left\{a_{i}\right\}$ for the known $L_{i}$ within the interpolation volume, and $\sigma_{\Delta}^{2}$, need to be determined for each scale,. Interpolation regions on the boundary of $L$, require coefficients consistent with the asymmetry of $L_{\Delta}$, i.e., the existence of finely scaled measured data on $L_{1}$ and $L_{2}$ or the lack of samples outside $L$. However, all regions away from the boundary use the same coefficients.

[24] Further interpolation to any temporal sampling period is possible using the method above. At finer temporal sampling, the log rain rate samples become more strongly correlated in time than in space. Temporal interpolation in the fine-scale limit can be performed by 1-D RMD independently at each spatial point.

[25] The ARMD algorithm has been extensively tested on numerically generated FBfs. It efficiently interpolates Gaussian FBfs and reproduces the expected statistics (i.e., the marginal distribution and covariance structure).

\subsection{Downscaling and Interpolation of CRIE Data}

[26] Radar derived rain rate fields have characteristics, such as intermittence and advection, and systematic errors that need to be addressed before interpolation and downscaling. Raw CRIE-derived rain rates are greater than a minimum $R_{\min }=0.05 \mathrm{~mm} / \mathrm{h}$, in all measurement voxels, due to random noise in the radar reflectivity measurements. Many applications treat measurements around the minimum to be zero rain rates. However, the $\mathrm{FBf} \log$ rain model is only applicable to areas where the rain rate is greater than zero, and the assumption of homogeneity cannot hold near the edges of rain fields. The marginal rain rate distribution is therefore assumed to have an atom at zero and otherwise to be lognormally distributed. The parameters of this hybrid distribution are estimated using Maximum Likelihood for each scan. The Maximum Likelihood algo- 


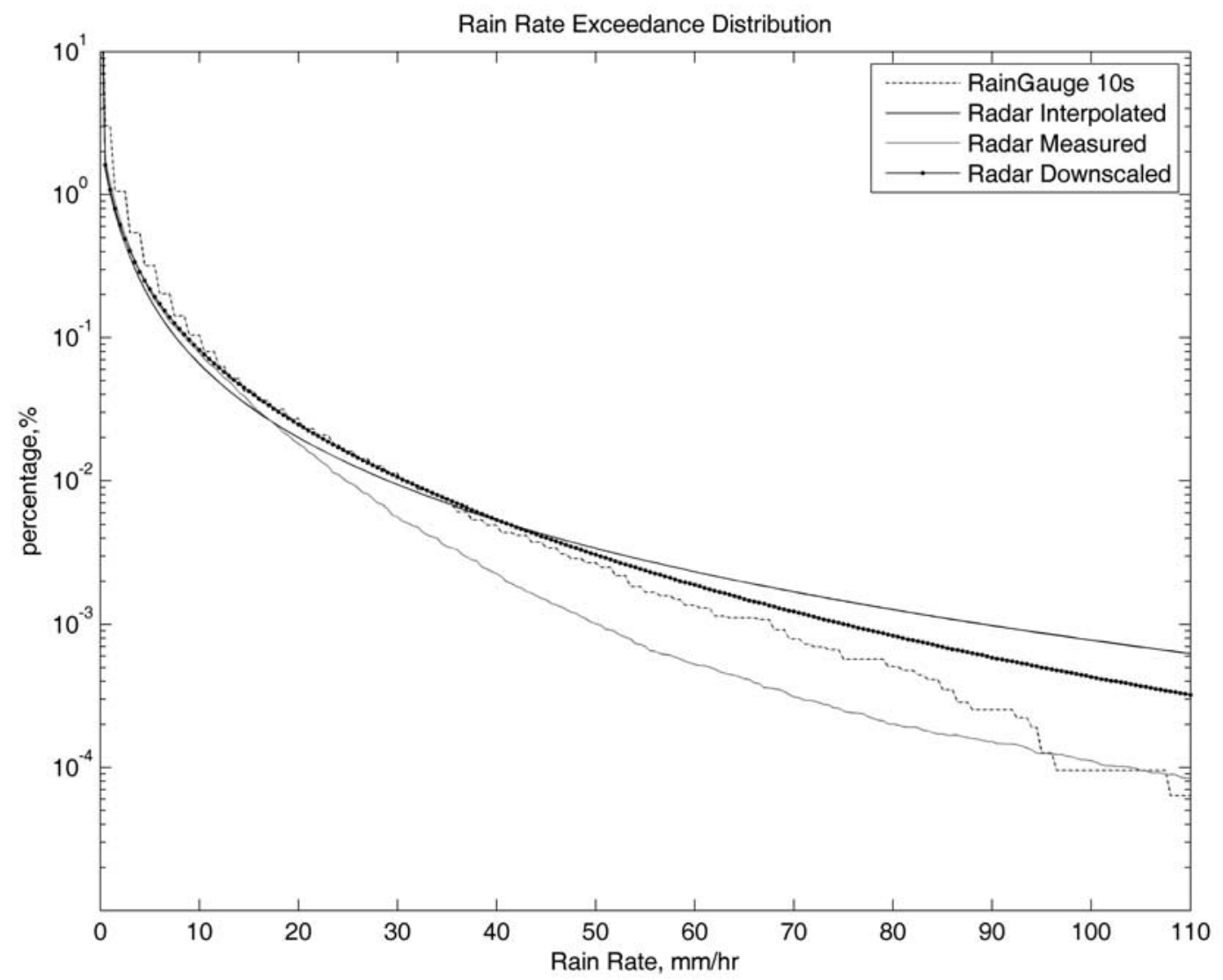

Figure 1. Comparison of rain rate exceedance distribution for original radar derived rain rate fields (light gray), downscaled data (black), interpolated-downscaled data (dark gray), and 9 gauge years of rapid response rain gauge data (dotted).

rithm allowed for the censorship imposed by radar measurement when determining the likelihood of each observation. The method was tested and verified using synthetic data with mean log rain rates corresponding to $1 \mathrm{~mm} / \mathrm{h}$. The interpolation mixes log rain rate data across event boundaries and so each measurement voxel must be assigned a nonzero rain rate. To achieve this, minimum curvature surfaces are fitted to measured log rain rate fields, i.e., surfaces are calculated that minimize the sum of the squares of the second partial derivatives and which pass through $\ln (R)$ where $R>R_{\min }$ and are constrained to be less than $\ln \left(R_{\min }\right)$ at all other points. This smoothly extrapolates log rain rates into areas of very low and zero rain rate and allows the multiscale downscaling algorithms to operate across rain event boundaries. Errors introduced by this approach will only affect the very low rain rates at the edges of events.

[27] The calculation of the downscaled and interpolated rain fields between two consecutive measured rain fields can be summarized by the following steps:
(1) Downscale by using Deidda's algorithm (section 3.1). (2) Estimate and remove the advection between the two measured rain rate fields, equation (2). (3) Calculate log rain rate over the analysis regions (section 3.3). (4) Calculate the relative spatial and temporal scales, i.e., maximize equation (6). (5) Interpolate scans using ARMD algorithm. (6) Reintroduce advection.

[28] In the following section, results are presented for downscaled and interpolated rain fields. Rain fields have been downscaled in three iterations of cascade by a factor of 8 to a spatial averaging over squares of side $\Delta_{x}=37.5 \mathrm{~m}$. Six iterations of the ARMD algorithm has then reduced the radar sampling time to $\Delta_{t} \cong 9.4 \mathrm{~s}$.

\section{Verification}

[29] Figure 1 shows the rain rate exceedance distributions of the original radar-derived rain fields, downscaled rain fields and the downscaled-interpolated fields. Downscaling does not conserve the distribution, and Figure 1 


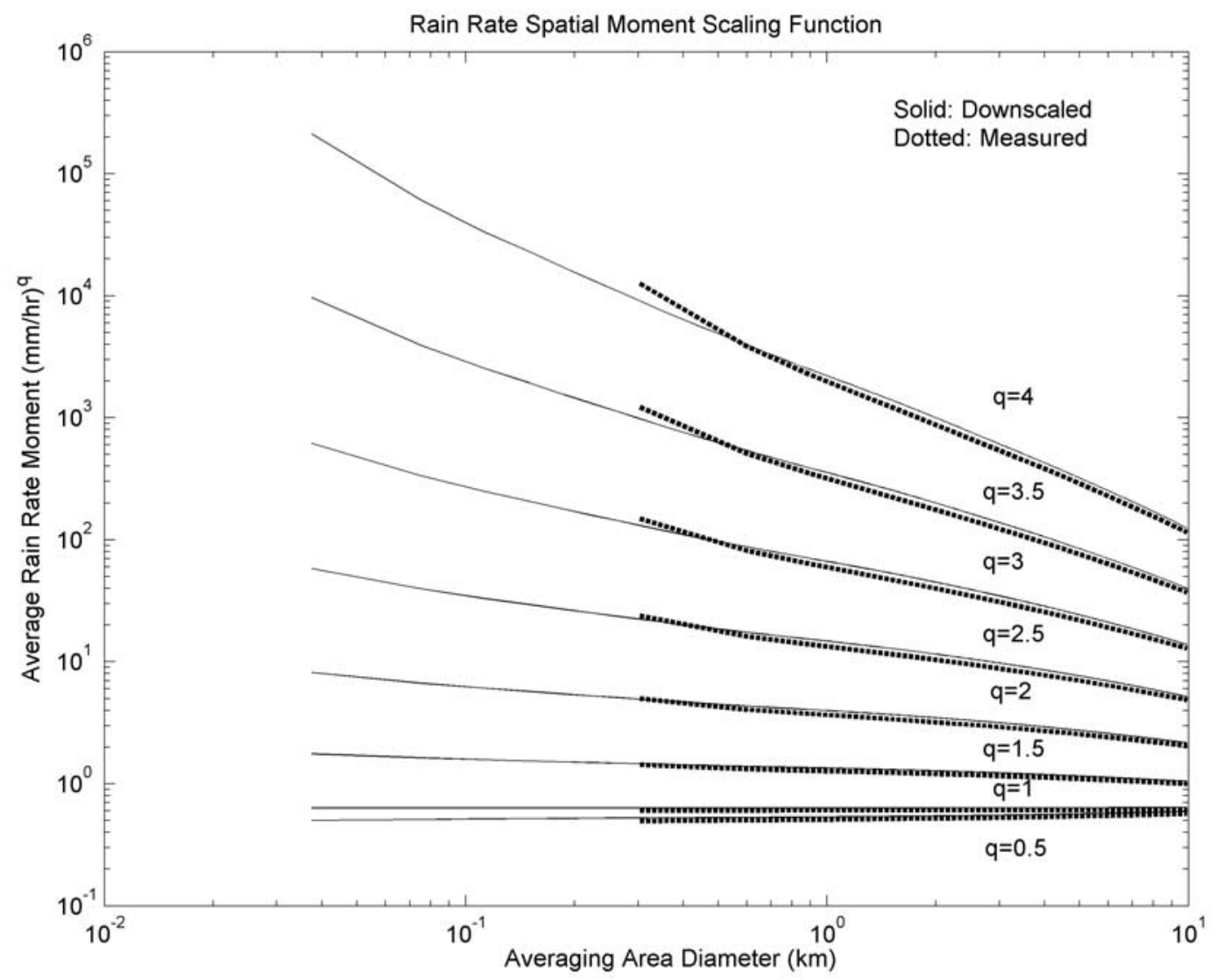

Figure 2. Spatial moment scaling function for the original radar derived rain rate fields (dotted) and the interpolated rain data (solid), for moments of order $q=0.5$ to $q=4$.

shows the increase in heavy rain rates introduced by downscaling. These three distributions are compared to the distribution of 9 gauge years of $10 \mathrm{~s}$, Chilbolton, rapid response rain gauge data [see Paulson, 2004], collected over the period 2000 to 2002 . The differences at low percentages of time are due to year-to-year variability.

[30] Interpolation aims to conserve the distribution after downscaling but this process has also increased the proportion of heavy rain rates. This is due to scans with multimodal $\log$ rain rate distributions. The mean and variance are poor summarizing statistics for these scans and, although these are conserved, interpolation has changed the distribution. This is a failing of the method, and the assumption of spatial homogeneity will be relaxed in future developments. This is particularly important if larger areas are interpolated as the scans are more likely to contain a mixture of stratiform and convective events. The current implementation reproduces the rain rate distribution up to the important $0.01 \%$ outage threshold.
[31] Figure 2 compares the spatial moment scaling function $S_{q}(\lambda)$ for the original radar derived rain fields and for the interpolated fields. Results for averaging regions with diameters below $300 \mathrm{~m}$ have been introduced by downscaling. Downscaling has extrapolated these statistics in a plausible way. The strong agreement between these two results is evidence that the interpolation method has preserved the spatial statistics of rain rate variation.

[32] Each spatial point yields a time series from which the temporal moment scaling function can be calculated. Figure 3 illustrates the temporal moment scaling function for the interpolated and downscaled rain rate data compared with 9 gauge years of rain rate measurements. Despite the radar and rain gauge data being acquired over different years, there is remarkable agreement between the two results. For an integration period of $10 \mathrm{~s}$, the moments for $1 \leq q \leq 2.5$ are indistinguishable on this plot implying that interpolated rain rates match measured statistics up to second order at least. It is difficult to quantify the year-to-year variability in 


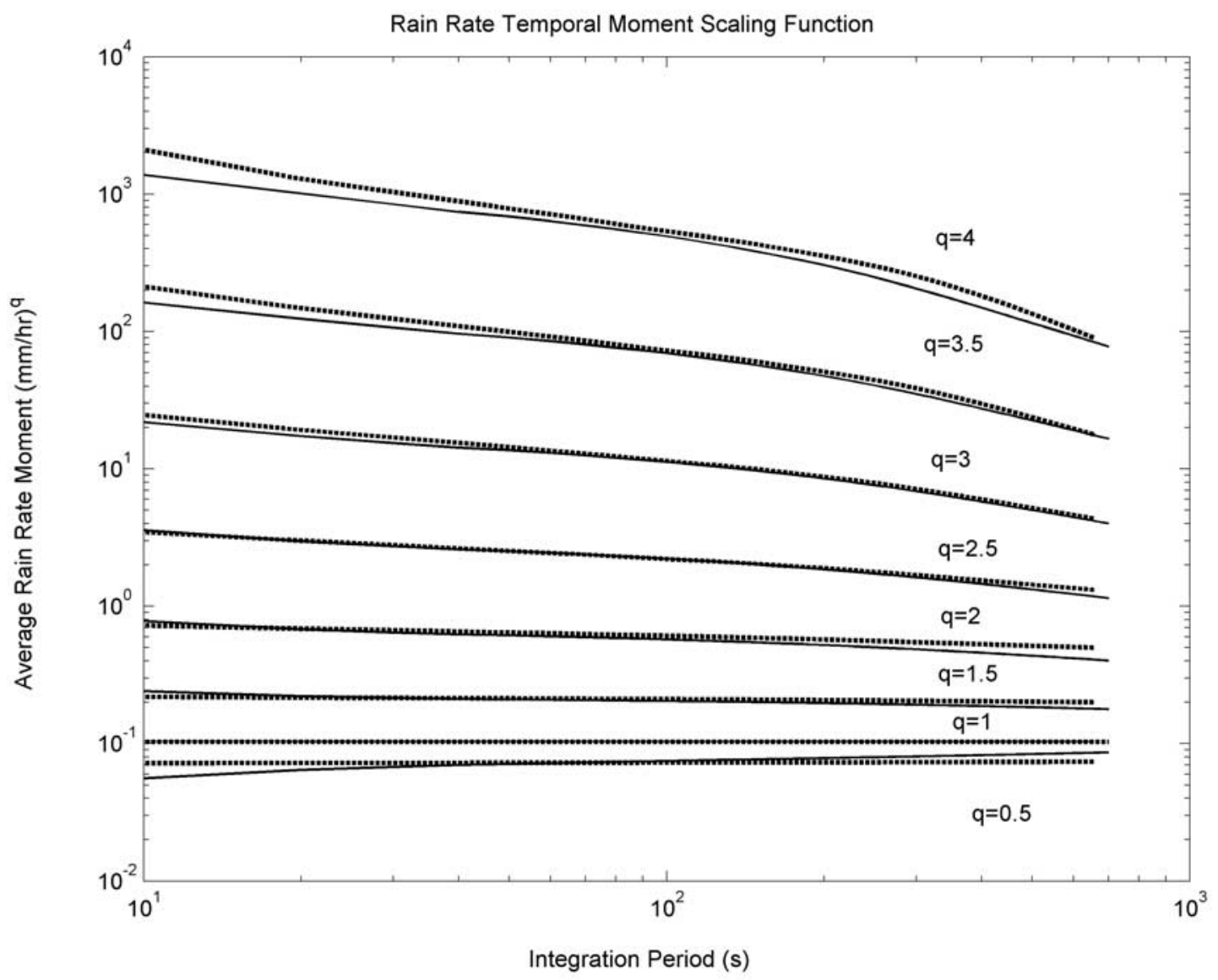

Figure 3. Temporal moment scaling function for 9 gauge years of RAL rapid response rain gauge data (solid) and downscaled-interpolated radar data (dotted).

summarizing statistics of rain rate and so it is similarly difficult to tell if the differences between higher-order moments are significant.

[33] The objective of the research has been to generate rain field time series as an input into a general microwave link rain fade simulation tool. To test the ability of the system to predict temporal variation, we have simulated rain fade time series of a single $38 \mathrm{GHz}, 9 \mathrm{~km}$ link and compared the derived fade duration model to the RAL model [Paulson and Gibbins, 2000; ITU-R, 2007a] (see Figure 4). The RAL model was derived from a 2-year fade time series measured on a $9 \mathrm{~km} 38 \mathrm{GHz}$ link at Chilbolton. It fitted lognormal curves to measured duration-frequency data for durations longer than $30 \mathrm{~s}$. The RAL model provides no information on the expected variation in the number of events that would be expected in a measured year. However, a reasonable assumption is that the variance is close to the mean number of events. Given this, the variation between simulated and model predictions is largely within the expected range, over the duration range that the RAL model is applicable. The effects of year-to-year variability could lead to the larger variation observed at low fade levels where rain events can produce different numbers of fade events depending on temporal variation of fade around the threshold level.

\section{Conclusions}

[34] A method has been developed to interpolate and downscale time series of rain fields measured by rain radar with interscan periods as long as $10 \mathrm{~min}$ to yield time series with sample periods as short as $10 \mathrm{~s}$. The method has been demonstrated using a database of radar derived rain fields from CAMRa, at Chilbolton in the UK. Interpolation has been shown to preserve the rain rate distribution and moment scaling of spatially averaged rain rate data. Furthermore, the interpolated and downscaled data have reproduced the moment scaling of 




Figure 4. The RAL fade duration model predictions for a $38 \mathrm{GHz}, 9 \mathrm{~km}$ link (solid) compared to the fade durations of a simulated link using downscaled and interpolated radar data (dotted). The curves indicate number of events in an average year for attenuations of $8,12,16,20,24$, and $28 \mathrm{~dB}$, from top to bottom.

temporally averaged rain rate data measured using rain gauges. The method has been applied to simulate time series of rain fade on a $38 \mathrm{GHz}$ link, and it has reproduced measured fade duration statistics. These rain rate field time series can be used to calculate joint rain fade time series for arbitrary networks of microwave links with an integration period as short as $10 \mathrm{~s}$. Further downscaling to $1 \mathrm{~s}$ will be justified when the data and statistical models are available.

\section{Notation}

$B_{L}(\mathbf{x}, \tau)$ Spatial-temporal second moment of log rain rate.

$\Delta_{x}, \Delta_{t} \quad$ Spatial averaging diameter and temporal sampling interval.

$L(\mathbf{x}, t) \quad$ Log rain rate over specified integration volume.

$R(\mathbf{x}, t) \quad$ Rain rate over specified integration volume.
$R_{\text {min }}$ Minimum rain rate defined by radar noise.

$E(\bullet) \quad$ Expected value.

$H$ Hurst coefficient.

$N\left(\mu, \sigma^{2}\right) \quad$ A normal distribution with mean $\mu$ and variance $\sigma^{2}$.

$S_{q}(\lambda) \quad q$ th order moment of rain rate averaged over volume of diameter $\lambda$.

$\varepsilon \quad$ An i.i.d sample from a standard normal distribution, e.g., $\varepsilon \in N(0,1)$.

$\xi(q) \quad q$ th order scaling exponent.

[35] Acknowledgments. We would like to acknowledge the support of the Radio Communications Research Unit of Rutherford Appleton Laboratory, and the staff of Chilbolton Observatory who acquired the radar data.

\section{References}

Callaghan, S. A. (2004), Fractal analysis and synthesis of rain fields for radio communication systems, Ph.D. thesis, Univ. of Portsmouth, Portsmouth, U.K. 
Callaghan, S. A. (2007), Model to generate synthetic rain intensity fields for any given area, ITU-R Doc., 3J/181-E, 3M/205-E.

Callaghan, S. A., and E. Vilar (2007), Fractal generation of rain fields: Synthetic realisation for radio communication systems, Microwaves Antennas Propag., 1(6), 1204-1211.

COST 210 Management Committee (1991), Influence of the atmosphere on interference between radio communications systems at frequencies above $1 \mathrm{GHz}, \operatorname{COST} 210$, Eur. Comm., Brussels.

COST 235 Management Committee (1996), Radiowave propagation effects on next-generation fixed-services terrestrial telecommunications systems, COST 235, Eur. Comm., Brussels.

COST 280 Management Committee (2005), Propagation impairment mitigation for millimeter wave radio systems, COST 280, Eur. Comm., Brussels, (Available at http://www.cost280.rl. ac.uk/).

Deidda, R. (1999), Multifractal analysis and simulation of rainfall fields in space, Phys. Chem. Earth, 24(1-2), 73-78.

Deidda, R. (2000), Rainfall downscaling in a space-time multifractal framework, Water Resour. Res., 36, 1779-1794.

Fenton, G. A., and E. Vanmarcke (1990), Simulation of random fields via local average subdivision, J. Eng. Mech., 116(8), $1733-1749$.

Goddard, J. W. F., and S. M. Cherry (1984), The ability of dualpolarisation radar (copolar linear) to predict rainfall rate and microwave attenuation, Radio Sci., 19(1), 201-208.

Goddard, J. W. F., and M. Thurai (1996), Modelling of attenuation due to rain on terrestrial paths using Chilbolton radar data, NRPP Res. Note 159, Rutherford Appleton Lab., Didcot, U.K.

Goddard, J. W. F., and M. Thurai (1997), Radar-derived path reduction factors for terrestrial systems, paper presented at Tenth International Conference on Antennas and Propagation, Inst. of Electr. Eng., Edinburgh, U.K.

Goddard, J. W. F., J. D. Eastment, and M. Thurai (1994), The Chilbolton advanced meteorological radar: A tool for multidisciplinary atmospheric research, Electron. Commun. Eng. J., $6(2), 77-86$.

ITU-R (2003a), Specific attenuation model for rain for use in prediction methods, ITU-R Recomm., P.838-2.

ITU-R (2003b), Propagation data and prediction methods required for the design of terrestrial broadband millimeter radio access systems operating in a frequency range of about 20-50 GHz in vegetation, ITU-R Recomm., P.1410-2.

ITU-R (2007a), Propagation data and prediction methods required for the design of terrestrial line-of-sight systems, ITU-R Recomm., P.530-12.

ITU-R (2007b), Characteristics of precipitation for propagation modelling, ITU-R Recomm., P.837-5, P Ser., parts 1 and 2, 2000, suppl. 1 .
Leitao, M. J., and P. A. Watson (1984), Application of dual linearly polarized radar data to prediction of microwave path attenuation at 10-30 GHz, Radio Sci., 19, 209-221.

Lovejoy, S., and D. Schertzer (2006), Stereophotography of rain drops and compound poisson-Cascade processes, paper presented at Cloud Conference, Am. Meteorol. Soc., Madison, Wis., $12-14$ July.

Mandelbrot, B. B., and J. W. Van Ness (1968), Fractional Brownian motion, fractional noises and applications, SIAM Rev., 10(4), 422-437.

Monin, A. S., and A. M. Yaglom (1971), Statistical Fluid Mechanics, vol. 1, MIT Press, Cambridge, Mass.

Monin, A. S., and A. M. Yaglom (1975), Statistical Fluid Mechanics, vol. 2, MIT Press, Cambridge, Mass.

Paulson, K. S. (2002), Spatial-temporal statistics of rain rate random fields, Radio Sci., 37(5), 1088, doi:10.1029/ 2001 RS002527.

Paulson, K. S. (2003), Prediction of diversity statistics on terrestrial microwave links, Radio Sci., 38(3), 1047, doi:10.1029/ 2001 RS002547.

Paulson, K. S. (2004), Fractal interpolation of rain rate time series, J. Geophys. Res., 109, D22102, doi:10.1029/2004JD004717.

Paulson, K. S., and C. J. Gibbins (2000), Rain models for the prediction of fade durations at millimeter wavelengths, IEE Proc., Part H, 147(6), 431-436.

Paulson, K. S., and X. Zhang (2007), Estimating the scaling of rain rate moments from radar and rain gauge, J. Geophys. Res., 112, D20107, doi:10.1029/2007JD008547.

She, Z.-S., and E. C. Waymire (1995), Quantised energy cascade and log-Poisson statistics in fully developed turbulence, Phys. Rev. Lett., 74(2), 262-265.

Tan, J., and L. Pedersen (2000), Study of simultaneous coverage and route diversity improvement under rainy periods for LMDS systems at $42 \mathrm{GHz}$, paper presented at Millenium Conference on Antennas and Propagation (AP2000), Eur. Space Agency, Davos, Switzerland, 9-14 April .

Usman, I. S. (2005), Development of point to multipoint models for availability and fade mitigation in the millimeter wave frequency range, Ph.D. thesis, Univ. of Bath, Bath, U.K.

Voss, R. F. (1985), Random fractal forgeries, in Fundamental Algorithms for Computer Graphics, edited by R. A. Earnshaw, NATO ASI Ser. F, Comput. Syst., vol. 17.

Wheater, H. S., V. S. Isham, C. Onof, R. E. Chandler, P. J. Northrop, P. Guiblin, S. M. Bate, D. Cox, and D. Koutsoyiannis (2000), Generation of spatially consistent rainfall data, Res. Rep. 204, Dep. of Stat. Sci. Univ. Coll. London, London, U.K.

Zawadzki, I. (1973), Statistical properties of precipitation patterns, J. Appl. Meteorol., 12, 459-472.

K. S. Paulson and X. Zhang, Department of Engineering, University of Hull, Kingston upon Hull HU6 7RX, UK. (k.paulson@hull.ac.uk) 